\title{
GESTIÓN DEL BOSQUE MEDITERRÁNEO Y CONSERVACIÓN DE LA AVIFAUNA: EL CASO DE LOS ALCORNOCALES
}

\author{
Carles BARRIOCANAL - Josep VILA \\ Departament de Geografia. Institut de Medi Ambient. Universitat de Girona
}

Recibido: 08/04/2011

Devuelto: $24 / 05 / 2011$

Aceptado: 27/07/2011

RESUMEN: Se presentan los resultados de los diversos estudios de poblaciones de aves realizados en los alcornocales catalanes, especialmente en cuanto a la riqueza específica de los mismos. Dado que los estudios publicados hasta el momento en esta materia eran referidos a la estación reproductora (primavera), se señalan los datos recogidos en los alcornocales de les Gavarres (NE peninsular), durante la estación invernal. Asimismo, se analizan las diversas tipologías de alcornocales que se encuentran en Catalunya, bosques en gestión activa, y por tanto sin sotobosque, y bosques abandonados en los que la biomasa del sotobosque es máxima, y se exponen los resultados de la comparativa entre ambas tipologías de explotación cuando se aplica un índice de conservación de la avifauna, basado en la riqueza de cada una de las especies registradas, la presencia de especies amenazadas según los criterios de la UICN (SPEC) y su categoría de amenaza (non-SPEC, SPEC 4, SPEC 3, SPEC 2 y SPEC 1). Los resultados obtenidos muestran como las explotaciones abandonadas tienen un índice de conservación de avifauna más elevado que los bosques en explotación activa. Se discuten estos resultados en el contexto de la gestión forestal y las ayudas recibidas por parte de las administraciones para llevar a cabo estas prácticas de gestión.

PALABRAS CLAVE: alcornocales, avifauna, gestión.

\section{MANAGEMENT OF MEDITERRANEAN FORESTS AND AVIAN CONSERVA-} TION: THE CASE OF LOS ALCORNOCALES

ABSTRACT: We present the results of the diverse studies of populations of birds realized in the Catalan cork oaks, especially for the specific richness. Provided that the studies published up to the moment in this matter were recounted to the breeding station (spring), we present the information gathered in the cork oaks of Gavarres (peninsular NE), during the winter station. Likewise, the diverse typologies of cork oaks that are in Catalonia, forests in active management and therefore without understory and forests left in that the biomass of the understory are maximum, are analyzed. We present the results of the comparative between both typologies of exploitation when there is applied an index of conservation of the avifauna, based on the richness of each of the registered species, the presence of species threatened according to the criteria of the UICN (SPEC) and his category of threat (non-SPEC, SPEC 3, SPEC 2 and SPEC 1). The obtained results show as the left exploitations have an index of conservation of avifauna higher than the forests in active exploitation. These results are dis- 
cussed in the context of the forest management and the grants received by the administrations to carry out these practices of management.

KEY WORDS: cork oak forests, avifauna, management.

\section{INTRODUCCIÓN}

Las comunidades de aves forestales están determinadas por un gran número de factores que incluyen la posición geográfica de los bosques, las características de los ambientes circundantes, el estadio de desarrollo de los árboles y su estructura y la composición de las especies (WIENS, 1989). Los bosques mediterráneos han estado, tradicionalmente, expuestos a una intensa presión humana mediante actividades como la silvicultura, la producción de carbón o el clareo de la vegetación para el pastoreo (BLONDEL y ARONSON, 1999). Sin embargo, los recientes cambios socioeconómicos acaecidos en las regiones desarrolladas están conduciendo a una reducción de estas actividades forestales, con la consiguiente acumulación de vegetación, biomasa susceptible de generar incendios forestales. En este contexto, la eliminación y reducción de biomasa de los ecosistemas forestales está siendo una de las principales actividades que se están llevando a cabo en los bosques de muchas regiones, especialmente en la cuenca Mediterránea occidental. El clareo mecánico del sotobosque para reducir las densas capas de arbustos y lianas acumuladas tiene como objetivo principal minimizar la carga de combustible, aunque con la misma práctica también se controla la competencia que podrían generar otras especies forestales, se favorece la regeneración de plántulas y se mejora el acceso a los bosques (MESÓN y MONTOYA, 1993). La influencia de la gestión forestal, del fuego y de los pastos en la diversidad biológica de las zonas arbustivas, garrigas y maquias del mundo Mediterráneo, ha sido ampliamente estudiada (BLONDEL, 1981; PRODON, 1988; PONS, 1998), pero no así los efectos del clareo del sotobosque (CAMPRODON y BROTONS, 2006). En ningún caso se han incorporado criterios de conservación de biodiversidad en los planes de manejo forestales. El clareo del sotobosque tiene un fuerte impacto en la biodiversidad dado que conlleva una significante eliminación de la biomasa forestal. En los bosques de la cuenca Mediterránea, más del $40 \%$ de la riqueza florística total y buena parte de la biomasa de artrópodos, recursos clave para diversos grupos faunísticos, se localizan en las capas arbustivas (CAMPRODON y BROTONS, 2006). La biomasa vegetal suele ser un buen indicador de la densidad y composición específica de las aves en los hábitats forestales (JAMES y WAMER, 1982). DONALD et $A L$. (1997) y DíAZ et $A L$. (1998) encontraron buenas correlaciones entre riqueza y densidad de aves y extensión del sotobosque. Todos estos estudios ponen de manifiesto que una intensa modificación de la capa arbustiva, tanto mecánicamente como mediante el uso de las quemas prescritas, de 
los ecosistemas forestales, tiene un significativo impacto en la avifauna. La desaparición del sotobosque en los ecosistemas forestales afecta a las aves de diversas formas: reducción de recursos tróficos (artrópodos y frutos), pérdida de lugares de canto y reproducción y refugio contra predadores, y cambios a nivel microclimático, que afectará a aquellas especies hidrofílicas como el caso del chochín Troglodytes troglodytes, el petirrojo Erithacus rubecula y la curruca capirotada Sylvia atricapilla. De hecho, los microclimas se han considerado como uno de los factores abióticos que determinan la distribución de las aves (WIENS, 1989). La reducción del sotobosque es una práctica forestal ampliamente extendida, pero mantener una gran cantidad de masa forestal en estas circunstancias requiere un esfuerzo que se traduce en unos enormes costes económicos.

Para mejorar la biodiversidad de las comunidades orníticas de un ecosistema forestal, se deberían asegurar un cierto número de áreas (patches) con presencia de arbustos y lianas. En el caso del encinar, especie próxima al alcornoque, CAMPRODON y BROTONS (2006) han calculado que para las especies del grupo Sylvia, después de una intervención de clareado forestal, se debería dejar un 30-40\% de superficie con la capa arbustiva con una altura de entre 1,5 y 2 metros. Al margen de la conservación de estas especies, la actuación propuesta conlleva además la protección de plántulas y la prevención de erosión del suelo.

\section{LOS ALCORNOCALES}

El alcornocal es una formación boscosa esclerófila que no sobrepasa los veinte metros de altura. En general, y como la mayoría de los bosques esclerófilos, dentro del sotobosque se identifican varios estratos arbustivos y el estrato herbáceo está poco desarrollado (NUET et AL., 1991). La sombra que produce el alcornocal no crea un verdadero ambiente nemoral y por lo tanto aparece un rico sotobosque tolerante a la luz. Para algunos autores, el alcornocal es la última de las grandes variantes del encinar litoral (FOLCH, 1981). La formación se sitúa en la alianza Quercion ilicis con una subasociación propia: Carici oedipostylae - Quercetum suberis. La distribución del alcornocal en el mundo comprende, en el sector situado más al oeste del Paleártico Occidental, desde la latitud de Córcega y Túnez al este, hasta el sur de Portugal y montañas del Atlas al oeste. Su distribución es discontinua y este hecho se explica por la gran variabilidad de los factores ambientales de esta extensa área y por la explotación antrópica que esta comunidad ha soportado en el tiempo y que ha modificado el patrón de distribución espacial (HAGGET, 1964). 


\section{FACTORES DE DISTRIBUCIÓN DEL ALCORNOCAL}

El clima óptimo para los alcornocales es aquel en el que la precipitación anual oscila entre los 500 y los $1.200 \mathrm{~mm}$. A pesar de este amplio rango pluviométrico, la comunidad puede soportar un cierto estrés hídrico estival. El rango termométrico se mueve entre los $-0,8$ y los $10,2^{\circ} \mathrm{C}$ de media mínima (m) y los 19,3 y $36,2^{\circ} \mathrm{C}$ de media máxima (M) (Pons, 1991). El sustrato en que los alcornocales se encuentran bien representados puede ser diverso pero nunca se detectarán en sectores calcáreos ni demasiado arcillosos. Se localizan principalmente en aquellos lugares silíceos donde el suelo es pobre en iones nutritivos y es de carácter marcadamente ácido (FOLCH, 1981). En general destaca su presencia sobre rocas plutónicas, metamórficas y paleozoicas, principalmente en granitos, granodioritas, gneis y esquistos. El límite altitudinal depende de la posición latitudinal en el conjunto de la distribución; en Marruecos, los alcornocales pueden llegar a encontrarse hasta los 2.000 metros de altitud (SAUVAGE, 1963), mientras que en Argelia el límite se sitúa en los 1.550 metros. En Andalucía los encontramos hasta 1.350 metros y en Catalunya se localizan, de forma natural, en los 600 metros en las solanas (VILAR et AL., 1989). En cuanto a los límites más bajos, hay que indicar que en el sur de Portugal se encuentran extensos alcornocales por debajo de los 200 metros. A pesar de no disponer de datos de la ocupación del alcornocal en el conjunto de su distribución mundial, en la Península Ibérica se detectan grandes montes al sur de Portugal, sur de Andalucía y en el nordeste de Catalunya. En conjunto ocupan aproximadamente medio millón de hectáreas. En Catalunya, una aproximación de la ocupación nos la dan ALLUÉ y MORENO (1989) que indican que había sido de unas 60.000 hectáreas, a pesar de que NATIVIDADE (1956) apunta a unas 80.000 has. a principios de siglo. VILAR et AL. (1989) apuntan, según memorias de producción, un paso de 46.000 has. a mediados de siglo, hasta un valor de 28.000 has. a mediados de los años 80. Lo que constatan estos autores es que la reducción de estos montes ha sido originada por los incendios forestales, la urbanización y la repoblación forestal con especies alóctonas (pinos, eucaliptos). En conjunto se calcula que actualmente en el conjunto del estado español hay 500.000 hectáreas de alcornocales (MONTOYA, 1988) a pesar de que el autor indica que quizás estos datos hayan sido sobredimensionados.

\section{ESTUDIOS DE LA AVIFAUNA DE LOS ALCORNOCALES}

Recientemente han aparecido estudios de análisis de los factores que afectan a la riqueza (valor promedio del número de especies contactadas) de aves en alcornocales marroquíes (CHERKAOUI et $A L$., 2009), en los que se demuestra que la diversidad y abundancia de árboles y arbustos determina la riqueza avifaunística. En la Península Ibérica los estudios de LEAL et $A L$. 
(2011) muestran cómo afecta la extracción de corcho a las poblaciones de aves, concluyendo que no hay diferencias entre bosques con extracción reciente y bosques con la capa de corcho en crecimiento. La afectación de aves coloniales en alcornoques centenarios ha sido estudiado por GARCía et $A L$. (2011) en las "pajareras" de Doñana, concluyendo que las aves influían en la salinidad del suelo y en consecuencia en la muerte de los alcornoques que sustentan las colonias. PONS (1991) ha estudiado la avifauna nidificante de los alcornocales del Mediterráneo occidental concluyendo que son las formaciones forestales paleártico-occidentales más ricas en cuanto a riqueza ornitológica, siendo las dehesas del centro de la península el subtipo de alcornocal con más diversidad ornítica debido a la coexistencia de especies forestales y no forestales.

\section{LOS ESTUDIOS EN CATALUNYA}

En Catalunya, la avifauna de los alcornocales ha sido ampliamente estudiada (PONS, 1991; BARRIOCANAL, 2003). En estos estudios se constata que la riqueza de especies es elevada (CUADRO 1), comparada con otras comunidades forestales como en el caso de los encinares; acercándose incluso a los valores que se pueden obtener en los bosques de ribera (BLONDEL, 1975). Las diferencias de riqueza entre los dos estudios -es 12,7 en PONS (1991) y 8,8 en BARRIOCANAL (2003)- pueden ser debidas a diversos factores como la diferencia entre el número de estaciones entre los dos trabajos (142 versus 60), la incorporación del Montseny en PONS (1991) por la posible influencia en los alcornocales de este macizo por la diversidad de hábitats forestales diferentes que se registran cerca de estos, y por la diferencia de estructura del hábitat forestal dado que los valores obtenidos en el trabajo de PONS (1991) fueron obtenidos en los años 80, veinte años antes que en el estudio de BARRIOCANAL (2003). BARRIOCANAL y SALVÀ (2004) estudiaron la riqueza de los alcornocales de la sierra litoral catalana (Macizo del Montnegre) y los valores obtenidos fueron similares a los de BARRIOCANAL (2003) para Gavarres, Cadiretes y Alberes (CUADRO 1). Ver MAPA 1 para la localización de los macizos referenciados.

Cuadro 1. Valores de riqueza de avifauna de los alcornocales catalanes

\begin{tabular}{lccl}
\hline \multicolumn{1}{c}{ Localización } & Riqueza & $n$ & \multicolumn{1}{c}{ Cita } \\
\hline Gavarres, Cadiretes y Alberes & 8,7 & 60 & Barriocanal (2003) \\
Gavarres, Alberes y Montseny & 12,7 & 142 & Pons (1991) \\
Montnegre & 8,15 & 20 & Barriocanal y Salvà (2004) \\
\hline
\end{tabular}

Este conjunto de trabajos muestran los valores de riqueza para la época reproductiva, momento más intenso de uso de los recursos que ofrece el alcornocal. BARRIOCANAL (2006) ha estudiado las poblaciones invernales en el 
macizo de las Gavarres, que muestran valores inferiores a los de la estación primaveral para este macizo (CUADRO 2).

Cuadro 2. Valores de riqueza de avifauna de los alcornocales de Les Gavarres en primavera y en invierno

\begin{tabular}{cccccc}
\hline Localización & Estación & Riqueza & Desv. Est. & $n$ & Cita \\
\hline Gavarres & Primavera & 8,8 & 2,17 & 20 & Barriocanal (2003) \\
Gavarres & Invierno & 5,65 & 1,76 & 20 & Barriocanal (2006) \\
\hline
\end{tabular}

\section{GESTIÓN DE LOS ALCORNOCALES}

Secularmente el hombre ha cultivado o favorecido al alcornoque para el aprovechamiento de su corteza con el fin de obtener corcho, producto usado principalmente para los tapones de las botellas de vino y cava. A lo largo de los siglos XIX y XX la industria del corcho era próspera y la gestión de los alcornocales comportaba un conjunto de actividades asociadas a su explotación, como el clareado del sotobosque con el objetivo de minimizar potenciales competidores por los recursos. En las explotaciones corcheras españolas, el siglo XX fue crítico y desde el periodo de la Guerra Civil el precio del corcho empezó a disminuir, en parte por la competencia del plástico en algunas de las aplicaciones típicamente corcheras. A partir de mediados de siglo, las explotaciones dejaron de ser rentables y se fueron abandonando. En consecuencia, los alcornocales se fueron densificando y el crecimiento del sotobosque fue tan intenso que alcanzó la altura de los árboles. Así, en un corto periodo de tiempo se pasó a una fisionomía del bosque totalmente diferente. A partir de la última década del siglo XX hay un cambio de tendencia y los precios del corcho aumentan. En Catalunya se reactivan algunas explotaciones dado que paralelamente al repunte del precio, seguramente por el efecto 2000 (el cambio de milenio hizo que se consumiera mucho vino y cava y por tanto la demanda de tapones de corcho fue elevada), el Departament d'Agricultura de la Generalitat de Catalunya destina recursos de los PDR (Planes de Desarrollo Rural), fondos de la Unión Europea, a la gestión de los alcornocales, abriendo algunas líneas de ayuda a las actividades en explotaciones de alcornoques, en las que toman especial relevancia las actuaciones de desbroce y eliminación del sotobosque. A partir de ese momento, la fisionomía de algunas explotaciones de alcornoque catalanas cambia y se da paso a una serie de fincas sin sotobosque, que se mezclan con aquellas que se mantienen abandonadas.

\section{VII. ÍNDICES DE CONSERVACIÓN DE LA AVIFAUNA}

La valoración de hábitats utilizando las aves es una práctica extendida, ya que este grupo es fácil de monitorear con un coste mínimo, existe un buen conocimiento de su biología y, al estar situados en la parte más elevada de 
las cadenas tróficas, integran los cambios de los niveles inferiores. Uno de los sistemas empleados es el uso de índices de conservación (PONS et $A L$., 2003). Estos índices de conservación integran, para cada especie, un valor de conservación Europeo según la clasificación de BirdLife International (TUCKER y HEAT, 1994), que utiliza el valor de SPECs (Categories of Species of European Conservation Concern) de la UICN (Unión Internacional para la Conservación de la Naturaleza). Estas categorías, cuantitativas, definen límites por debajo de los cuales aumenta el grado de amenaza de una especie y están basados en aspectos vinculados al tamaño poblacional, la extensión del área de distribución y las tendencias temporales actuales, así como sus proyecciones futuras (CARRASCAL y PALOMINO, 2006). Estas categorías se definen según la amenaza de cada especie: Non-SPEC, SPEC 4, SPEC 3, SPEC 2, SPEC 1. La última categoría, SPEC 1 es la que corresponde a las más amenazadas (especie globalmente amenazada); las categorías SPEC 2 y 3 se consideran con un estatus de conservación desfavorable a escala Europea, aunque SPEC 3 muestra especies que no se localizan exclusivamente en el continente Europeo. SPEC4 corresponde al grupo de especies no amenazadas solamente a nivel Europeo (pueden estarlo en otros continentes). Finalmente, non-SPEC es el conjunto de especies que no se encuentran amenazadas ni a nivel Europeo ni a nivel mundial.

Combinando los datos de riqueza de aves de una comunidad y la presencia de especies amenazadas según las categorías de la UICN, se puede obtener un valor de índice de conservación. La fórmula más adecuada sería la que proponen Pons et AL. (2003):

$$
-\sum^{\mathrm{k}}{ }_{\mathrm{i}=1}=\left[\log \left(\mathrm{A}_{\mathrm{i}}+1\right) \text { X SPEC value }{ }_{\mathrm{i}}\right]
$$

donde $\mathrm{k}$ es la riqueza de especies, $\mathrm{A}_{\mathrm{i}}$ la frecuencia relativa y SPEC value, el valor de SPEC de cada una de les especies detectadas, que se ha calculado en una progresión aritmética a medida que se incrementa el valor de conservación siguiendo el siguiente criterio: $\mathrm{NONSPEC}=1, \mathrm{SPEC} 4=2, \mathrm{SPEC} 3=4$, $\mathrm{SPEC} 2=8, \mathrm{SPEC} 1=16$. Así, se obtiene un índice de conservación de la avifauna, que cuanto más elevado sea el valor resultante, más presencia de especies amenazadas o sensibles, a nivel europeo. Si se comparan los índices de conservación de avifauna entre dos sistemas de manejo para una misma tipología de bosques, se puede valorar el uso de un determinado sistema de gestión más adecuado para la conservación de las aves.

\section{INDICES DE CONSERVACIÓN DE LAS AVES DE LOS ALCORNOCALES: UN ESTUDIO EN LES GAVARRES}

Los alcornocales del macizo de les Gavarres (NE peninsular) ofrecen un 
adecuado marco para la realización de un estudio de conservación de avifauna en función de la tipología de los manejos del bosque. Una localización de parcelas sin sotobosque, producto de la gestión forestal y otras sin gestionar, en las que el sotobosque es tan elevado como el propio bosque, permitió llevar a cabo una serie de censos en los que se determinó la riqueza avifaunística de cada una de las tipologías de explotación, y, aplicando la fórmula del índice de conservación, se valoró qué método de gestión es más adecuado para las poblaciones de aves en cuanto a conservación. Se localizaron un total de 20 parcelas para cada tipología (con gestión o abandonadas) de los alcornocales del macizo de les Gavarres. Este número de parcelas de muestreo es el adecuado para obtener resultados contrastables en ambientes forestales (BLONDEL, et AL., 1970). A continuación se determinó la riqueza promedio de cada una de las tipologías de parcelas (para la metodología utilizada ver el trabajo de BARRIOCANAL (2003)). Con estos datos, y aplicando el índice de conservación para cada una de las especies censadas, se pudo realizar el análisis del valor de conservación para cada tipología de alcornocales. Tanto en el caso de los alcornocales gestionados, y por tanto sin sotobosque, como los abandonados, aparecieron especies SPEC 2 y SPEC3 (CUADRO 3), es decir, especies con estatus de conservación desfavorable a escala Europea. En las parcelas sin sotobosque se registró la totovía común (Lullula arbórea), especie que necesita zonas abiertas para su alimentación y la tórtola común (Streptopelia turtur). En las parcelas cerradas con sotobosque, las especies amenazadas a nivel Europeo que se citaron fueron el herrerillo capuchino (Parus cristatus), el pito real (Picus viridis), el mosquitero papialbo (Phylloscopus bonelli) y, nuevamente, la tórtola común. La aplicación del índice de conservación, la fórmula que considera la riqueza de las especies y su estado de conservación Europeo, dio como resultado que, en conjunto, las parcelas con sotobosque tienen un valor de conservación mayor $(44,09)$ que las gestionadas $(34,95)$.

\section{CONCLUSIONES}

Acorde con los estudios de aves llevados a cabo en los alcornocales del mediterráneo occidental, estos ambientes son los que disponen de una diversidad avifaunística más elevada (PONS, 1991; BARRIOCANAL, 2003; BARRIOCANAL y SALVÀ, 2004). En la actualidad, la gestión de estos bosques depende del precio del corcho y de las ayudas de las administraciones, por lo que se pueden encontrar diversas tipologías de explotación del alcornocal que, como se ha visto, pueden influir notablemente en la composición de la diversidad avifaunística. A nivel de conservación, ambos tipos de explotación presentan especies categorizadas como amenazadas según los criterios de la UICN. A pesar que PALOMINO y CARRASCAL (2006) apuntan que estos 
criterios tienden a tener un sesgo político-geográfico, categorizando especies con elevado grado de amenaza si son escasas en países centro europeos e Inglaterra, y que estas aproximaciones globales pueden no ser de utilidad a escalas geográficas y políticas menores, consideramos que en este estudio son adecuados, ya que buena parte de las especies clasificadas como SPEC 2 y 3 , también están amenazadas a nivel regional, según los datos del Atles dels ocells nidificants de Catalunya (ESTRADA et AL., 2004).

Cuadro 3. Especies de aves amenazadas registradas en los censos de los alcornocales de les gavarres en función de la tipología de explotación

\begin{tabular}{ccc}
\hline \multicolumn{2}{l}{ Especies presentes en las parcelas con sotobosque } & \\
\hline Especies & Frecuencia & Cat. conservación \\
\hline Streptopelia turtur & $35 \%$ & SPEC 3 \\
Parus cristatus & $15 \%$ & SPEC 2 \\
Phylloscopus bonelli & $5 \%$ & SPEC 2 \\
Picus viridis & $5 \%$ & SPEC 2
\end{tabular}

Especies presentes en las parcelas sin sotobosque

\begin{tabular}{lcl}
\hline \multicolumn{1}{c}{ Especies } & Frecuencia & Cat. conservación \\
\hline Lullula arborea & $35 \%$ & SPEC 2 \\
Streptopelia turtur & $5 \%$ & SPEC 3 \\
\hline
\end{tabular}

Los resultados muestran como los bosques de alcornocales abandonados sostienen unas poblaciones avifaunísticas con más interés de conservación que los gestionados. Cabe indicar que solamente una especie amenazada (Tórtola común, SPEC 3) aparece en las dos tipologías de explotación, y cada una de ellas sostiene otras especies también amenazadas. Esto nos indica que para mantener y conservar especies con un status de conservación sensible, y a pesar que los alcornocales abandonados han tenido un valor de conservación más elevado, cabe mantener ambas tipologías de explotaciones para de esta manera asegurar la conservación de todas las especies registradas que se encuentran amenazadas. Así, el escenario ideal, seria disponer de bosques en explotación activa junto con otros abandonados, con una elevada densidad de sotobosque. De hecho, en la actualidad, esta situación es la que se produce en el macizo de les Gavarres y en los de Cadiretes y Alberes, donde también hay alcornocales. Esta situación, ideal ahora mismo para mantener las especies amenazadas, es altamente inestable, ya que si suprimen las líneas de ayuda a la extracción de corcho o bien aparecen ayudas para el fomento de otras especies forestales, los propietarios podrían, en el primer caso, abandonar las explotaciones, con la consiguiente aparición del, con el tiempo, denso sotobosque, o cambiar totalmente las explotaciones, llegando a desaparecer los alcornocales y las especies que estos abrigan. $\mathrm{Pa}$ ra que esto no llegue a suceder, las administraciones deberían hacerse eco de la importancia biológica de los alcornocales y trabajar junto con los gestores 
en su conservación. Esto incluirían subvenciones, subsidios agroecológicos a partir del Programa de Desarrollo Rural (PDR) de la Unión Europea, y fórmulas como las deducciones fiscales a cambio del compromiso de la propiedad de conservar el patrimonio natural, las ecotasas que se podrían obtener a partir de impuestos especiales o mediante las actividades turísticas en el medio natural (CAMPRODON, 2009). Para finalizar, se debería también tener en consideración los efectos del cambio climático sobre las especies forestales mediterráneas. Un estudio reciente de la Universidad de Extremadura (FELICÍSIMO et $A L ., 2011$ ), sitúa a los alcornocales de la mitad sur occidental de la Península Ibérica como una de las comunidades que más se verá afectada por los efectos del aumento de las temperaturas y la aridez, y apunta que tal vez esta podría llegar a desaparecer en Extremadura dada su necesidad de recursos hídricos frente otras especies como la encina (Quercus ilex).

\section{BIBLIOGRAFÍA}

Allue, M. y Montero, G. (1989): «Aportaciones al conocimiento fitoclimático de los alcornocales catalanes». Scientia Gerundensis, ${ }^{\circ} 15,161-178$.

BARRIOCANAL, C. (2003): Anàlisi de l'ornitofauna dels boscos i bosquines esclerofil.les de les muntanyes de la Costa Brava. Tesis doctoral. Universitat de Barcelona.

BARRIOCANAL, C y SALVÀ, M. (2004): «Caracterització de l'avifauna de les suredes del Montnegre a la primavera: comparació amb les suredes gironines». Ponencia en $I V$ Trobada d'Estudiosos del Montnegre i el Corredor. Barcelona, Diputació de Barcelona, 123-128.

BARRIOCANAL, C. (2006): «Explotación del alcornocal y poblaciones de aves: un estudio en les Gavarres (NE peninsular)». Ponencia en III Congreso Español de Biogeografia, Comunicaciones. Vitoria, Universidad del País Vasco, 343-347.

BLONDEL, J. FERRY, C. y FrochOT, B. (1970): «La méthode des indices ponctuels d'abondance (IPA) ou des relevés d'avifauna par "stations d'écoute"». Alauda $\mathrm{n}^{\circ}$ $38,55-71$.

BLONDEL, J. (1975): «L'analyse des peuplements d'oiseaux, elements d'un diagnostic ecologique I. La methode des Echantillonages Frequentiels Progressifs (E.F.P.)». Terre et Vie, $\mathrm{n}^{\circ} 29$, 533-589.

BLONDEL, J. (1981): «Structure and dynamics of bird communities in Mediterranean habitats» en Mediterranean-Type shrublands (di Castri, F.; Goodall, D.W.; Specht, R.L., eds.). Amsterdam, Elsevier, 361-385.

BLONDEL, J. y ARONSON, J. (1999): Biology and Wildlife of the Mediterranean Region. Oxford University Press, Oxford.

CAMPRODON, J. y BROTONS, L. (2006): «Effects of undergrowth clearing on the bird communities of the Northestern Mediterranean Coppice Holm oak forests». Forest Ecology and Management, $\mathrm{n}^{\circ} 221,72-82$.

CAmprodon, J. (2009): «Biodiversitat i gestió forestal: estat de la qüestió». Medi Ambient. Tecnologia i Cultura, $\mathrm{n}^{\circ}$ 44, 7-12. 
CARrascal, J.M. y PAlomino, D. (2006): «Rareza, estatus de conservación y sus determinantes ecológicos. Revisión de su aplicación a escala regional». Graellsia, $\mathrm{n}^{\mathrm{o}}$ 62, 523-538.

Cherkaoui, I., Slaheddine, S., Jihen, B. Rguibi-IDrissi, H. y DaKKi, M. (2009): «Factors affecting bird richness in a fragmented cork oak forest in Morocco». Acta Oecologica, no 35 (2), 197-205.

Díaz, M., Carbonell, R. SAntos, T. y Tellería, J.L. (1998): «Breeding bird communities in pine plantations of the Spanish plateau: biogeography, landscape and vegetation effects». Journal of Applied Ecology, n 35(4), 562-574.

DONALD, P.F., HAYCOCK, D. y FULLER, R.J. (1997): «Winter birds communities in forest plantation in western England and their response to vegetation, growth stage and grazing». Bird Study, no 44(2), 206-219.

Estrada, J., Pedrocchi, V., Brotons. L. y Herrando, S.(eds.) (2004): Atles dels ocells nidificants de Catalunya 1999-2002. Institut Català d'Ornitologia (ICO)/Lynx Edicions, Barcelona.

Felicísimo, A., Villalba, C., Mateo, R.G. y MuÑOZ, J. (2011) Vulnerabilidad y adaptación de la Biodiversidad Española frente al cambio climático. 1 Flora y vegetación. Ministerio de Medio Ambiente y Medio Rural y Marino.

FolCH, R. (1981): La Vegetació dels Països Catalans. Ketres Editora. Barcelona.

Garcia, L.V., Ramo, C., Aponte, A., Moreno, A., Dominguez, M.T., GomezAPARICIO, L. REDONDO, R. y MARAÑ́n, T. (2011): «Protected wading bird species threaten relict centenarian cork oaks in a Mediterranean Biosphere Reserve: A conservation management conflict» Biological Conservation, $\mathrm{n}^{\circ} 144,764-771$.

HAGGET, P. (1964): «Towards a statistical definition of ecological range: the case of Quercus suber». Ecology, n 45, 622-625.

JAMES, F.C. y WAMER, N.O. (1982): «Relationships between temperate forest bird communities and vegetation structure». Ecology, n 63, 159-171.

LEAL, A.I., CORREIA, R.A., GRANADEIRO, J.P. y PALMEIRIM, J.M. (2011): »Impact of cork extraction on birds: Relevance for conservation of Mediterranean biodiversity» Biological Conservation (in press).

Mesón, M. y Montoya, M. (1993): Selvicultura Mediterránea. Mundi-prensa, Madrid.

MonTOYA, J.M.( 1988): Los alcornocales. Ed. SEA, Madrid.

NATIVIDADE, J. (1956): Subericulture. Ecole Nationale des Eaux et Forets. Nancy.

NueT, J., PANAREDA, J.M. y Romo A.M. (1991): Vegetació de Catalunya. Editorial Eumo.

PONS, P. (1991): Biogeografia i ecologia de l'avifauna nidificant en les suredes de la Mediterrània occidental. Tesina, Universitat de Barcelona.

PONS, P. (1998): «Bird site tenacity after prescribed burning in a Mediterranean scrubland» en Fire Management and Landscape Ecology (Trabaud, L., ed.) Washington, International Association of Wildland Fire, 261-270.

Pons, P., LAmbert, B., Rigolot, E. y Prodon, R. (2003): «The effects of grassland management using fire on habitat occupancy and conservation of birds in a mosaic landscape». Biodiversity and Conservation, $\mathrm{n}^{\circ} 12$, 1843-1860. 
Prodon, R. (1988): Dynamique des systems avifauna-végétation après déprise rurales et incendies dans le Pyrénées méditerranéennes siliceuses. Thèse de Doctorat de Etat. Univesité Paris 6.

Sauvage, C. (1963): Atlas du Maroc. Comité National de Géographie du Maroc. Rabat.

TUCKER, G.M. y HEAT, M.F. (1994): Birds in Europe: their conservation status. BirdLife Conservation series 3. Birdlife International, Cambridge, UK.

Vilar, L., PolO, L. y Domínguez-Planella, A. (1989): «Los alcornocales de la provincia de Girona». Scientia Gerundensis, n $15,143-151$.

WiEns, J.A. (1989): The Ecology of Bird Communities. Cambridge University Press, Cambridge.

Mapa 1. Distribución de los alcornoques en Catalunya.

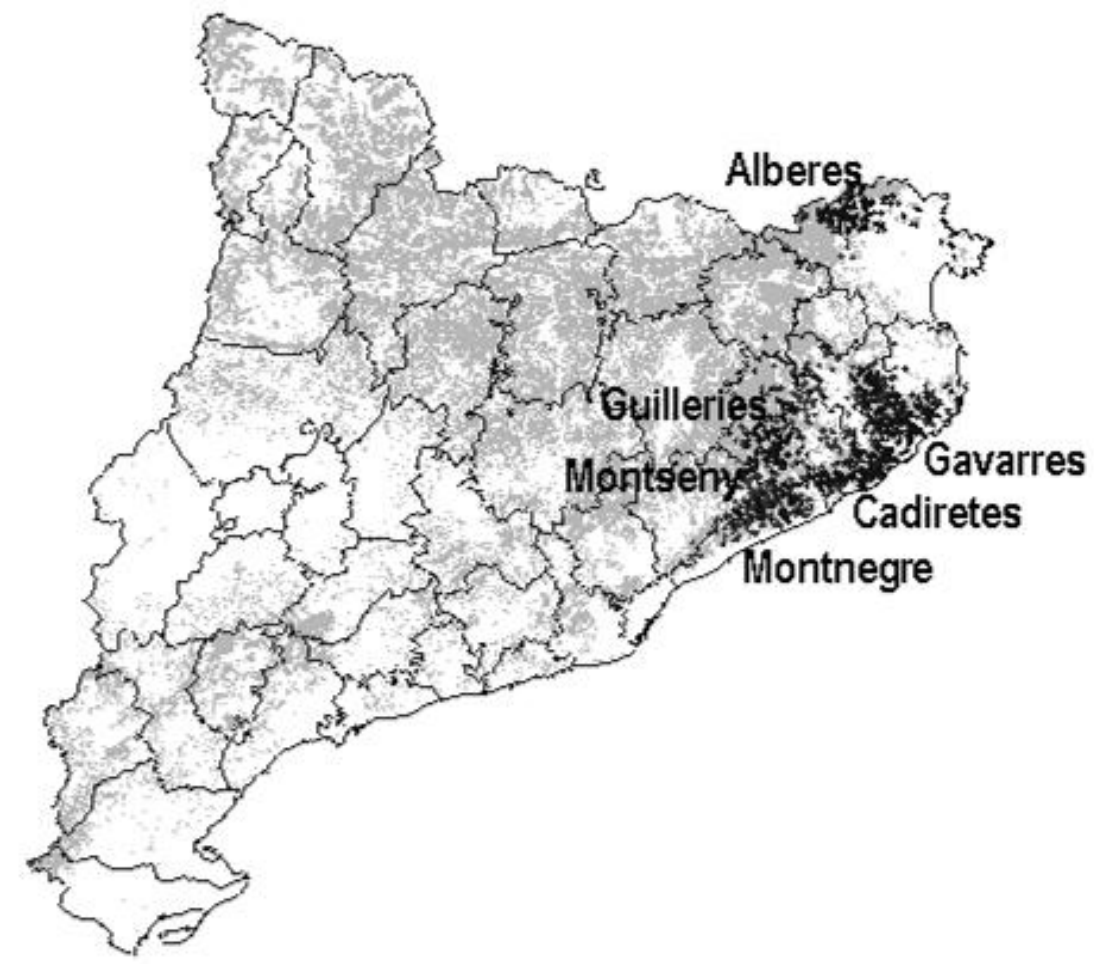

FUENTE: elaboración propia a partir del mapa de distribución del CREAF (http://www.creaf.uab.es/iefc/pub/introduccio/especies/mapasurera.htm). 